Journal of Engineering and Applied Sciences 12 (16): 4267-4283, 2017

ISSN: 1816-949X

(C) Medwell Journals, 2017

\title{
A Review of Genetic Algorithm Applications in Solving Vehicle Routing Problem
}

\author{
${ }^{1,}{ }^{4}$ Mazin Abed Mohammed, ${ }^{1}$ Mohd Khanapi Abd Ghani, ${ }^{2}$ Omar Ibrahim Obaid, \\ ${ }^{3}$ Salama A. Mostafa, ${ }^{3}$ Mohd Sharifuddin Ahmad, \\ ${ }^{4}$ Dheyaa Ahmed Ibrahim and ${ }^{1}$ M.A. Burhanuddin \\ ${ }^{1}$ Biomedical Computing and Engineering Technologies (BIOCORE) Applied Research Group, \\ Faculty of Information and Communication Technology, \\ Universiti Teknikal Malaysia Melaka, Melaka, Malaysia \\ ${ }^{2}$ College of Education, Al-Iraqia University, Adhamyia, Baghdad, Iraq \\ ${ }^{3}$ Faculty of Computer Science and Information Technology, Universiti Tun Hussein Onn Malaysia, \\ Johor, Malaysia \\ ${ }^{4}$ DepartmentPlanning and Follow Up, University of Anbar, Ramadi, Iraq
}

\begin{abstract}
Vehicle Routing Problem (VRP) has been considered as an important part in logistics dealing. Thus, an appropriate chosen of vehicle routing plays a considerable factor to improve the economic interests and suitability of logistics projects. In this study, we review the recent work in one of the most traditional problems in logistics which is the VRP. The study manifests the objective of providing a clear and detailed discussion on applying genetic algorithm to the VRP to be optimally solved. It further presents many examples of applying genetic algorithm in finding optimal solutions to the VRP.
\end{abstract}

Key words: Vehicle Routing Problem (VRP), Capacitated Vehicle Routing Problem (CVRP), genetic algorithm, Evolutionary Algorithm (EA), optimization, recent work

\section{INTRODUCTION}

The Vehicle Routing Problem (VRP) (Laporte, 1992; Toth and Vigo, 2002; Cortes et al., 2017) has been considered as an important factor in the domain of logistics. Thus an appropriate chosen of vehicle routing plays a considerable factor to improve the economic interests and suitability of logistics projects. In general, vehicle routing problem is identified as follows. Once the positions of all clients have been known, the routes of all vehicles should be specified with a view to ensure transportation routes or costs are the shortest or lowest.

The vehicle routing problem becomes an area of research since it was studied by Dantzig and Ramser in 1959. It has been investigated by many researchers for $>50$ years (Cortes et al., 2017). The concept of VRP can be portrayed as the related issues of resolving shortest path routes from one location to a set of geographically dispersed stations (customers, cities, universities, warehouses, schools, stores, etc.) (Toth and Vigo, 2014). The basic and traditional problem in routing which has been addressed by researchers for more than a hundred year is the Travelling Salesman Problem (TSP) who distributes goods to a group of cities and returns to his hometown (Laporte, 1992). The condition is that the salesman visits each city once on his routing trip provided that he follows the shortest and the least costly route. In fact, the salesman problem is an arithmetic problem that can be easily represented by a graph showing a collection of nodes that represent the cities to be visited. Many researchers dealt with this problem and they used almost all kinds of algorithms to find the optimal solution to it which to some extent succeeded in solving the problem when the number of cities was limited and failed when the number of cities was increased. Conventional vehicle routing problem is summarized as follows: a fleet of vehicles (for distribution of goods) starts from one location and visits a group of scattered cities or customers and return to the same location with minimum distance and costs on the conditions (Kovacs, 2008):

- Every city is visited by one vehicle only once within a single route

- The capacity of each vehicle is enough for all cities included in the route

Corresponding Author: Mazin Abed Mohammed, Biomedical Computing and Engineering Technologies (BIOCORE) Applied Research Group, Faculty of Information and Communication Technology,

Universiti Teknikal Malaysia Melaka, Melaka, Malaysia 
Routes begin and end at the same location. The number of vehicles is less than the proposed routes as well as the number of routes are less than that can be provided to cover all cities. Since, the presentation of VRP in 1959 by Dantzig and Ramser, it has stirred the enthusiasm of numerous researchers to study and solve this problem. Numerous algorithms and techniques have been enhanced for the VRP from exact algorithms to heuristic search and to metaheuristic. In classical heuristic studies, the aim was essentially to get an acceptable solution fast and subsequently improve the result. Other algorithms that have been used for this problem are the Jaikumar algorithms, the Sweep algorithm and the Fisher and saving algorithm. In modern search methods, many algorithms have been used and developed which depend on two fundamental standards: local search and population search. local search, the method moves starting with one solution to other with a group of nominee solutions until a best solution is found or the ideal opportunity for pursuit terminated. The good known methods of this kind are Bees Algorithm (BA), Simulated Annealing (SA), Harmony Search (HS) and Tabu Search (TS). In population search, a group of potential parent solutions are created and recombined through a many process (crossover, mutation, selection) to find practical and best results. The best methods of this kind are genetic algorithm and Adaptive Memory Procedures (AMPs) (Barbucha and Jedrzejowicz, 2007; Laporte, 2009).

The VRP problem is one of the known interesting issues that invited many concern and requested a lot of case studies because it deals with real cases that have many applications and it is not easy to find a best solution for it. Many approaches have been improved to resolve the VRP problem and search for good solutions, but for almost all smallest problems, finding the lowest cost function is not easy. These approaches can be divided into two groups: exact methods and non-exact methods which can in turn be divided into two sub-groups: Heuristic methods and Meta-Heuristic methods. Many algorithms have been used to deal with few or all variants of the problem to achieve optimality.

The vehicle routing problem: The vehicle routing problem is one of the difficult assignments in operation research field. It is a NP-hard improvement issue which implies that there is no quick method exists for its result (Braysy and Gendreau, 2001). This issue has numerous applications in our life: routing of salespeople, dial-a-ride systems, street cleaning, waste collection, school-bus routing, Transportation of handicapped people and Collection and delivery of goods. The problem can be described as a set

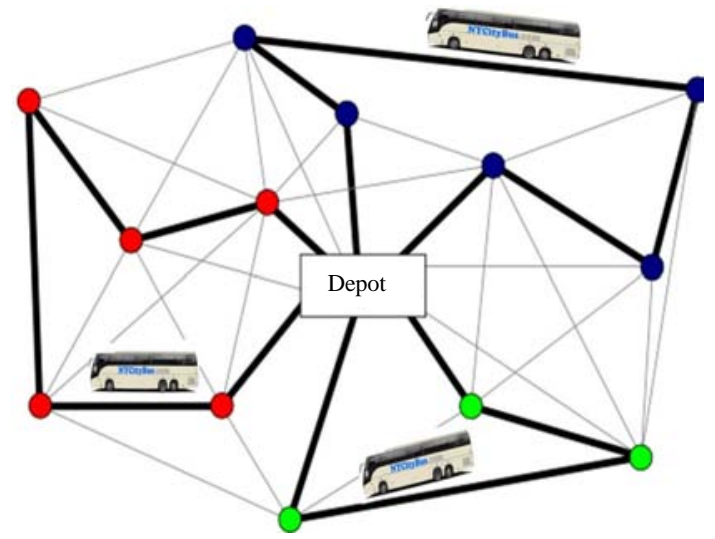

Fig. 1: An example of VRP

of vehicles that starts from a depot or more than one depot and has to serve a set of customers in which each vehicle has a limited capacity and each customer has to be visited once during each route (Fig. 1).

The vehicle routing problems have many different types according to variants of constraints, e.g., periodic VRP (PVRP), the Vehicle Routing Problem with Time Windows (VRPTW), the Multi-depot Vehicle Routing Problem (MDVRP), the Capacitated Vehicle Routing Problem (CVRP), Pickup and Delivery Vehicle Routing Problem (PDVRP), the Site Dependent Vehicle Routing Problem (SDVRP), the Open Vehicle Routing Problem (OVRP) and time-dependent VRP (TVRP). These problems have been studied intensively by many researchers. The best known problems are the VRPTW, CVRP and PDVRP (Braysy and Gendreau, 2001; Sen and Bulbul, 2008).

Both Exact and Heuristic methods have been used for solving the VRP. Some of the well-known heuristics include Miller's Sweep algorithm, gillett, wright's saving algorithm and clarke. Exact techniques such as branch and cut are also successful at solving the problem (Torres et al., 2009). Almost all types of metaheuristic have been used to solve the VRP such as Genetic Algorithms (GA), Deterministic Annealing (DA), Bees Algorithm (BA), Large Neighborhood Search (LNS), Fuzzy Logic (FL), Tabu Search (TS), Scatter Search (SS), Ant Systems (AS), Harmony Search (HS), Neural Networks (NN) and Simulated Annealing (SA) (Ropke, 2005; Eksioglu et al., 2009).

Vigo (2007) clarified that heuristics are capable of solving the VRP for optimality because they can build a feasible solution and at the same time minimize the cost to as low as possible. He added that the approaches easily handle the variety of constraints that arise in real world and should take into consideration the simplicity and flexibility in addition to accuracy and speed. 


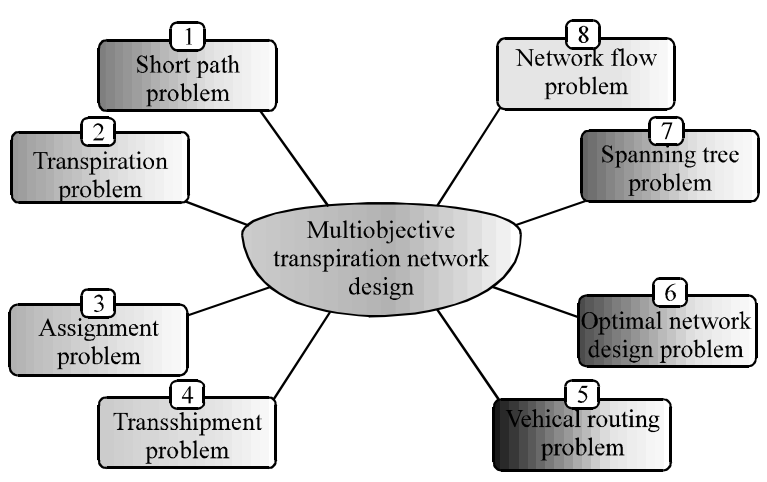

Fig. 2: A set of routing problem including VRP

Yeun described the classic VRP and the types of dynamic problem. They also reviewed all the algorithms used to solve this problem for optimality. They stated that heuristics searches such as genetic algorithm, tabu search, scatter search and simulated annealing could obtain best solutions with different types of problem. Many researchers now are interested in using Genetic Algorithm, Fuzzy Logic (FL) and Neural Network to solve the search problem.

VRP definition and background: The VRP can be described by the following graph-theoretic problem. Let $\mathrm{G}=(\mathrm{V}, \mathrm{A})$ be a complete graph where $\mathrm{V}=\{0,1, \ldots, \mathrm{n}\}$ is a vertex set corresponds to known non-negative demand (dj) customers and A is the arc set between the vertices where vertex 0 corresponds to the depot. A non-negative cost, cij is linked to each arc (i, j) $\in \mathrm{A}$ and represents the traveling cost from vertex $i$ to vertex $j$. If the cost values satisfy cij $=$ cji for all $\mathrm{i}, \mathrm{j} \in \mathrm{V}$, the problem is considered as symmetric VRP, otherwise, it is called asymmetric VRP. In many cases the cost matrix satisfies the triangle disparity, such that cik + ckj $=$ cij for any i, j, $\mathrm{k} \in \mathrm{V}$ (Zirour, 2008; Wang et al., 2009).

The VRP aims to find a collection of $\mathrm{k}$ simple circuits, each associated with a vehicle route with minimum cost which is considered as the costs sum of the arcs of circuits such that:

- The depot; vertex 0 is visited by each circuit

- Each vertex $\mathrm{j} \in \mathrm{V} \backslash\{0\}$ is visited only by one circuit

- The vehicle capacity $\mathrm{C}$ is not exceeded by the sum of the vertice's demand visited by a circuit

Figure 2 presents a set of routing problems including the VRP and provides borders isolating the VRP from others which helps researchers to know the form of the VRP and what is relations.
VRP in real life: The VRP has many applications in real life. It appears in a wide area of transportation and distribution systems such as transportation of people and products, delivery service and garbage collection. All these problems have economic importance, particularly in developed countries. The economic factor in saving expenditures is a strong motive for companies and researchers to find the best way to resolve and improve transport efficiency (Eksioglu et al., 2009).

Static VRP: Static Vehicle Routing Problem (SVRP) means that all the data on the route is known in advance before starting and does not change after the route began. The problem becomes dynamic if any restriction is imposed, like time, vehicle capacity or other variables (Barbucha and Jedrzejowicz, 2007; Wang et al., 2009). Exact algorithms, heuristic and metaheuristic are used to solve the VRP for optimality (Laporte, 2009). This is the traditional and basic VRP in which all the data on the routes (customer location and demands) is known in advance before starting and is unchanged or updated after the routes began (Zirour, 2008; Yueqin et al., 2007). This means no customers (cities) or new demands need to be inserted into one of the routes. In fact, if a new request has to be inserted, it becomes very complex and needs further planning process. If the capacities of all vehicles are identical and fixed, the problem is considered as the Capacitated VRP (CVRP).

Wang et al. (2009) utilized GA in solving the VRP and enhanced the mutation step and the coding plan of the method utilizing the normal numbers. They utilized the normal numbers for the chromosome which represents to the path. Consequently, they created the populations by using the crossover operations and in addition two processes of mutation. The main operator of mutation is enhanced inversion mutation process and the other is the quality trade mutation process. They utilized improvement keep methodology to enhance a strategic distance from the loss of the enhanced individual in which the best individual is utilized rather than the most exceedingly terrible one. Hence, they demonstrated that their enhanced GA abbreviated the coding length and improved the result proficiency.

Yueqin et al. (2007) applied GA to resolve the VRP issue with many limitations. They showed a new method (Finite Automaton) to create single population and propose a new technique edified by hermaphrodites. Each time a finite automaton starts; it produces a new population in every generation's evolution and then brings the good individuals to the next population until the individual population is constructed. They deemed the central station as a customer, since all paths begin and 
end on it. In the selection process they suggested another procedure that chooses from a similar individual to crossover, select sets of chromosomes from which the poorest quality in the chromosome is chosen and expelled indiscriminately from the chromosome and after that broken. The experimental results were reported to be good and encouraging for solving VRPs with multiple depots.

Dynamic VRP: Dynamic Vehicle Routing Problem (DVRP) means that not all the information on the routes is known in advance before starting (Feng et al., 2009). The problem is also dynamic if information is changed or updated or if any restriction added, like time window, vehicle capacity or other variables. This problem needs real time algorithm, since new request should be taken into consideration.

Goel and Gruhn (2006) presented a new algorithm that is based on large neighbourhood search to solve the dynamic vehicle routing problem. They used fast insertion methods for requests to achieve fast response. The first one is the sequential method in which all new requests are chosen and their possible insertions are determined. The other is the auction method in which all vehicles are sent out with a possibility and efficiency of insertion for new requests. Each request to be inserted is allocated to a vehicle with low cost to be inserted in its tour. They have proven that the algorithm performs well when the number of vehicles and transfer requests are high with less response time.

Montemanni et al. (2003) presented an algorithm for solving VRP using Ant Colony paradigm in which the DVRP is decomposed into a sequence of static VRPs. They proposed three main elements. The first is an event manager which records new orders. The second element is the Ant Colony algorithm which is based on the computational model inspired from real ant colonies function. The third is pheromone conservation in which a matrix including all the data for optimal solutions is used. If a static problem is similar to the next one, the information of good solution is passed on to the next problem. Their algorithm achieved good results compared with other heuristic techniques.

Rizzoli et al. (2004) discussed a VRP with all its constraints and various metaheuristics that have been applied to solveit. They described the ant colony optimization and how to solve the vrp with many types. They presented two real world applications of ant colony optimization with the time dependent VRP. They defined the Ant Colony optimization as one of the most effective meta-heuristics that can give optimal solution for VRP and their real world applications prove that.

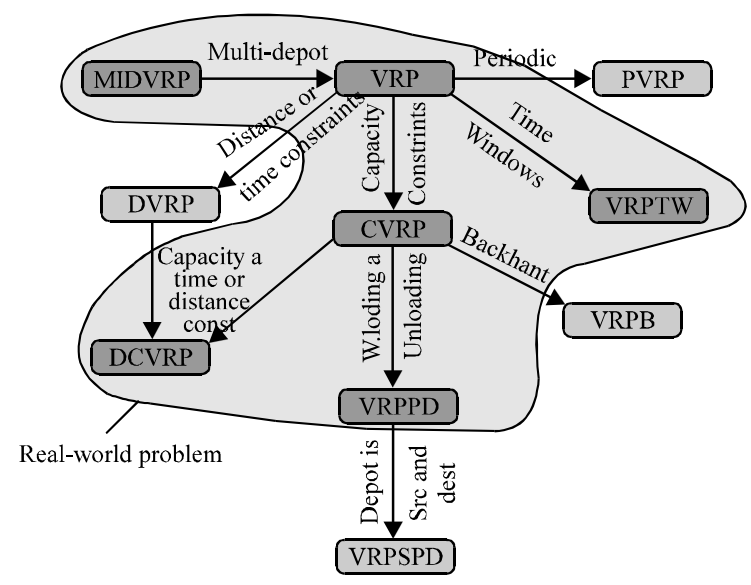

Fig. 3: VAP types

VRP types: The role of vehicle routing problem in distribution and logistics is very essential. Efforts carried out to study the VRP since Dantzig and Ramser have defined the problem as a generalized problem of Travelling Salesman Problem (TSP) in1959. Many researchers have described several VRP types such as Capacitated Vehicle Routing Problem (CVRP) Open Vehicle Routing Problem and Vehicle Routing Problem with Time Windows (VRPTW).

In this study, the main types of Vehicle Routing Problem are addressed in addition to the major research conducted to find appropriate solutions (Fig. 3).

Vehicle routing problem with time windows: The Vehicle Routing Problem with Time Windows (VRPTW) has been the target of extensive research and almost all types of meta-heuristic have been applied to solve the problem. In this type of vehicle routing problem, a time window constraint is considered in which each city or customer has a specific time to be serviced when the vehicle has to arrive. The VRPTW is represented by a fleet of similar vehicles symbolized by $\mathrm{V}$ and a directed graph, $\mathrm{G}$ which contains a set of vertices that represent the customers, $\mathrm{C}$. The nodes 0 and $n+1$ represent the depots, i.e., starting depot and returning depot respectively. The set of $n$ vertices representing customers is denoted by $\mathrm{N}$ (Obaid et al., 2015; Mostafa et al., 2012).

All possible connections between the nodes (including the nodes denoting depot) are denoted by the arc set $\mathrm{A}$. All routes start at 0 and end at $n+1$, no arc terminate at node 0 and no arc originates at node $n+1$. A $\operatorname{cost} \mathrm{Cij}$ and a time tij is associated with each arc $(i, j) \in \mathrm{A}$ of the routing network (Braysy and Gendreau, 2001). The travel time tij may comprise service time at customer $i$. Each vehicle has limited capacity $q$ and each customer $i$, has a demand di, $i \in \mathrm{C}$ and a time window [ai, bi] where ai 


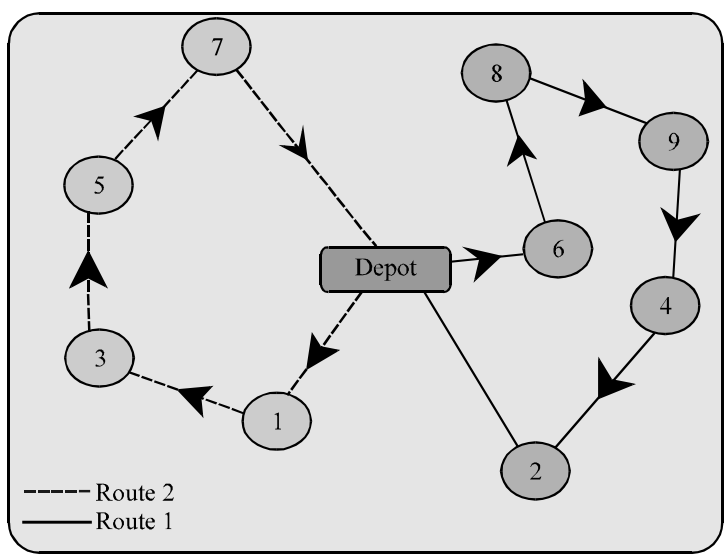

Fig. 4: Example of a routing solution for VRPTW

and bi are opening time and closing times of $i$, respectively. The probability of vehicle arrival before the beginning of the time window (i.e., ai) leads to waiting time until service is possible, while no vehicle should arrive after the given time interval, bi. Vehicles must also depart the depot within the depot time window $[\mathrm{a} 0, \mathrm{~b} 0]$ and must come back before or at time bn+1. Supposing that waiting time is allowed with no cost, it can be assumed that $\mathrm{a} 0=\mathrm{b} 0=0$; therefore, all routes start at time 0.

The goal of the VRPTW is to service all the C customers using the $\mathrm{V}$ vehicles such that the following objectives are met and the following constraints are satisfied (Braysy and Gendreau, 2001).

\section{Objectives:}

- Reduce the summation of vehicles numbers are utilized to service the customer

- Reduce the distance are travelled around by the vehicles

\section{Constraints:}

- Vehicle ability limitation is spotted

- Time window limitation must be spotted

- Every customer is serviced exactly once

- Each vehicle path begins at vertex 0 and ends at vertex $n+1$

Figure 4 shows a graphical model of the vehicle routing problem with time windows and its solution. Figure 4 shows two routes, Route 1 with 5 customers and Route 2 with 4 customers. Braysy and Gendreau (2001) used Genetic algorithm to solve the VRP with time windows in which there is a specific time for any customer to be served. Then, he made a comparison between his results and the results of other algorithms like Scatter
Search algorithm and Bees algorithm. He discovered that the genetic algorithm performance is inferior to the other algorithms.

Cordeau and Gendreau (2002) explored the Tabu Search heuristics which are used to solve the VRP and explained their features such as short term neighborhood structures and long term memory. They defined the tabu search as one of the most successful search that are used to solve VRP due to many ideas contained in many implementations of search such as the allowance of new parameters. The tabu search is adopted due its accuracy, speed, simplicity and flexibility. Pisinger and Ropke (2005) applied a heuristic technique that is able to solve VRP with five different constraints such as time window, capacity, multi-depot, site dependent and open vehicle routing problem. They used Adaptive Large Neighborhood Search (ALNS) which is an extension of the large neighborhood search framework by Shaw with an adaptive layer. They describe a framework which can be adapted for other problem types to define a number of good performance heuristics that can build the kernel of an ALNS Application. They discovered that the integration between good and poor heuristics form robust algorithm that gives best results rather than using one heuristics.

Csiszar (2005) presented route removal technique for VRP with time windows to minimize the number of routes and estimated cost. He presented two phase solutions: One for route elimination and the other for the cost reduction. The results showed that his algorithm is competitive against the best algorithms. Desaulniers et al. (2009) used a new algorithm to solve the problem with time windows by using a Large Neighborhood Search algorithm (LNS) based on heuristic branch-and-price method to explore the neighborhood using different procedures from another algorithm. LNS are frameworks that represent a number of simple neighborhoods completed to modify the current solution in a local search. They ranked their proposed algorithm within the top five algorithms which solved the problem with larger and more difficult instances. Azi et al. (2010) used an exact algorithm for the first time to solve the VRP with multiple use of vehicle with which each one executes many routes according to a time window and customer demands. They used the column generation algorithm with a branch-andprice algorithm to facilitate the problem formulation. The column generation algorithm is used when customers are chosen according to profit to be gained from them in case it is not possible to serve them all. The result shows that their method is limited by problem size which can be solved with 20 customers but many research problems have up to 40 customers. 
$\mathrm{Xu}$ et al. (2008) used genetic algorithm to solve the VRP with time windows and fuzzy demand to reduce the total distance for vehicles routes and the delay times at the customers due to time violations. The GA is applied to solve this problem which is formulated in two stages; the first stage reduces the cost in both stages due to initial solutions and in the second stage, the failure for cost of route. Their research shows that their solutions are close to the best solutions. Zhao et al. (2008) showed the dynamic VRP with time windows which considers the time delay caused by traffic congestion during a day. They considered soft time windows which allows vehicle delay but with penalty. Their system is efficient and more flexible to resolve their problem compared with other heuristics. They considered routes planning and the accidents that appeared during the day. Based on travel time model, the algorithm calculated the speed that reflects the traffic congestion. They show three different methods to obtain three routes of different types in which the range from slow too high for average speeds are created. They aimed to reduce the time spent by routes and the customers time delay. Their research proves that the variable speed model is durable and achieves better results compared with the constant speed model.

Nazif and Lee (2010) presented an optimized crossover operator in genetic algorithm application to solve the dynamic VRP with time window. Optimized crossover was applied by Aggarrwal et al. (1997)'s genetic algorithm (Mohammed et al., 2014). The crossover operator is the main factor to select individuals from a population for generating offspring. Instead of the traditional way to produce offspring they used the swap node operator which randomly selects and swaps two nodes from a parent and repeated on the second parent to create a second offspring. They test their results with other algorithms with good solutions and show that their results are competitive. Tang et al. (1996) studied the VRP with time windows (VRPTW) and proposed a mathematical model for it. He proceeded to solve the model by improved genetic algorithm. First, the initial population is computed then a pair of individuals is selected after computing the fitness values to make the crossover to generate two new individuals. He used a novel order crossover operator (NOX) which is better than other operators in generating a new child that is different from the parents. For mutation he used a modified mutation operator which includes swapping mutation and inversion operator. In swapping, the genes are swapped on two selected positions. In inversion operator, the chromosome is found randomly. Using two cutting points, the operation to produce the child by inverting the substring between them. The research results show that the improved Genetic algorithm is effective in achieving good solutions.

The capacitated VRP: The Capacitated Vehicle Routing Problem (CVRP) is the basic type of routing problem in which the vehicles have limited capacity. In the traditional CVRP, all information about customer or route demands is clear. The objective is to minimize the total travel cost. The CVRP is the same as the VRP if the total of all customer or traveler demands for each route or path do not exceed the vehicle's capacity. The VRP is considered dynamic if the capacity of each vehicle is not limited to take into consideration any new demand (Mohammed et al., 2015a, b).

Shaw (1998) used a Large Neighborhood Search (LNS) with constraint programming technology for solving capacitated vehicle routing problems. The technique of LNS acts like a local search in making moves, but uses a tree-based search with constraint to improve the cost and validity of the travel. The research shows that the average solutions produced by LNS and its performance are close and competitive to the best published solutions of the best operations research meta-heuristic methods. Baldacci et al. (2004) presented a new branch-and-cut algorithm to solve the CVRP. They derive a new lower bound using Linear Programming (LP) and compare with the lower bound of different CVRP formulations. Their research shows that their algorithm is capable of solving the problem for large instance.

Lopes et al. (2005) presented an Ant Colony algorithm (ACO) to solve the CVRP. They proposed two levels of optimization: the first level searches for desired routes and the second one optimizes each route as a travelling salesman problem. They showed that their research results are good for small instances. Kuske and Luderer ( 2010) built a model for an Ant Colony algorithm to resolve CVRP as a general public of autonomous units. They demonstrated the self-sufficient conduct of each ant as a self-sufficient unit which responds freely in a general situation while searching for an objective. Their outcome about demonstrate that the autonomous units improve ant colony optimization technique.

Bouhafs et al. (2010) used a Hybrid Heuristic technique to resolve the problem by combining an Ant Colony algorithm with a Savings algorithm. Their research results show that Hybrid Heuristic technique is competitive with the other approaches such as Scatter search, bees algorithm and simulated annealing. Mohammed et al. (2012) consider the utilization of a GA in resolve CVRP in which an group of vehicles with limits on capability and moving time are accessible to benefit an 
group of customers and compelled by soonest and most recent time for serving. The outcomes demonstrate that GA s eligible to decide the best path for the vehicles while keeping up their requirements of limit and travel time. Nowadays, evolutionary algorithms like Genetic algorithm and neural network are the main interests of many researchers. GA may not solve the problem and find an optimal solution or may lead to the dead end (Obaid et al., 2012) but it can find an acceptable or feasible solution better than other algorithms in reasonable time.

Multi-Depot VRP: In a Multi-Depot Vehicle Routing Problem (MDVRP), vehicles start their routes from several depots and return to them at the end of their routes. Minimization of all routes distances is the main objective in MDVRP. Polacek et al. (2004) presented a Variable Neighborhood Search (VNS) to solve MDVRP with time windows. VNS is a meta-heuristic method for solving optimization problems proposed by Mladenovic and Hansen in 1997. In this meta-heuristics and contrary to many local searches, several neighborhood structures are used instead of a single one. During descent and exploration phases, the change of neighborhood is applied to improve optimality. Their research showed that the results are competitive to an existing TS approach.

Parragh et al. (2008) presented adaptive Variable Neighborhood Search (VNS) to solve MDVRPTW by introducing two parallel approaches of VNS using two cooperation schemes. The first scheme stores and manages the best found solutions and the most important search parameters. The second one reproduces the successful features of the successive VNS. Their research results show that in 11 cases out of 20 , new best solutions are obtained. Carlsson et al. (2009) presented two heuristics to minimize the maximal distance of a route. The first one is a linear programming-based approach with global improvement to assign customers to depots and generate routes for each vehicle. The second one is the partition heuristic which divides the service region into equal sub-regions that contain the same number of nodes. Then they proposed a fast approximation algorithm to generate good initial solutions. Their research techniques give good results than conventional local search methods.

Lau et al. (2010) presented a new method to optimize vehicle routing problem with multiple depots, multiple customers and multiple products by using a new search technique called the Fuzzy Logic-guided Genetic Algorithms (FLGA). FL is used to adjust the crossover and mutation rates after ten consecutive generations. In crossover, a new crossover operator is used to solve this problem called the Partial Uniform and Partial Order (PUPO) crossover. The PUPO crossover includes two types of crossover: a uniform crossover and an order crossover. In mutation a new mutation operator is proposed to solve this problem called the Partial Uniform and a Partial Swap (PUPS) mutation. The PUPS mutation contains two types of mutation: a uniform mutation and a swap mutation. The results show that their search technique in a set of data generated randomly surpasses other searches used to solve the problem such as branch and bound, standard GA, Simulated Annealing and Tabu Search.

Vidal et al. (2012) presented a hybrid genetic algorithm to solve the multi-depot and periodic VRP. They proposed many contributions in crossover operation, management of unfeasible solutions, evaluation procedure and diversity. These contributions enhance the hybrid algorithm to achieve good solutions.

Open VRP: In the Open Vehicle Routing Problem (OVRP) a route ends after a vehicle visited the last city and it does not need to return to the depot it started from. If the vehicle needs to return to the depot, it must re-track the same route. Many algorithms have been used to solve this problem. Tarantilis and Kiranoudis used a meta-heuristic called the "list-based threshold accepting algorithm" to solve a multi-depot OVRP while tarantilis used the simulated annealing-based algorithm to solve the problem. Brandao proposed a Tabu Search algorithm for OVRP with a maximum route length constraint. $\mathrm{Li}$ presented a record-to-record travel heuristic, Pisinger and Ropke (2005) published an adaptive neighborhood search heuristic. Adam presented a branch-and-cut algorithm for the Capacitated Open Vehicle Routing Problem (COVRP). They show that their research results are good for small and medium scale instances of the COVRP.

Ozyurt et al. (2006) presented an enhanced parallel savings algorithm with Tabu Search and nearest insertion algorithm to solve open VRP with deadlines.

VRP with pickup and delivery: In the Vehicle Routing Problem with Pickup and Delivery (VRPPD) there are two locations for each customer in the same route, one for pickup and the other for delivery. The goods collected in the first location should be delivered to the second location (Desrochers et al., 1999, 1990). Thus, the problem could be divided into two independent CVRPs, one for delivery and the other for pickup (Mohammed et al., 2016a, b). Vehicle Routing Problem with Pickup and Delivery is also called VRP with backhauls (VRPB). Additionally, with a restriction that all goods must be delivered before any goods can be picked up, the last vehicle is and first to move out (LIFO) (Powell et al., 2001). 


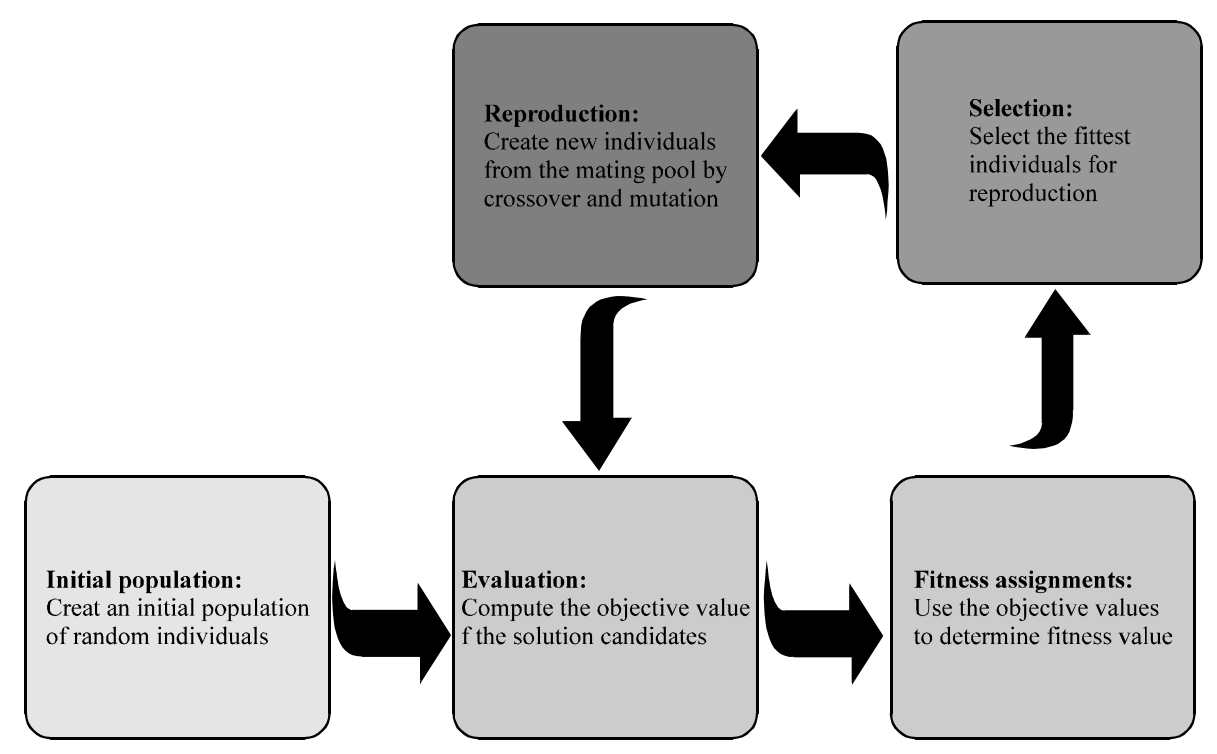

Fig. 5: The basic cycle of EA

Potter and Bossomaier (1995) studied the static VRP with Dial-A-Ride Problem (DARP) which is a special case of the General Pickup and Delivery Problem (GPDP) in which passengers in addition to goods, may need to be transported from one place to another. By reviewing the use of genetic algorithm they try to find a proximate optimum solution to the DARP in case of large numbers of passengers. They used a two-level genetic algorithm to solve this problem. In the upper level, the passengers are allocated to vehicles and in the lower level, the shortest path for the current number of salesman in each vehicle is found. The crossover operator applied in the study is moved matched crossover (PMX) proposed by Oliver, Smith and Holland which maintains the locations of units in the parents while switching between two cut points. Their results show that the genetic algorithm is effective in obtaining good and accurate solutions compared to other methods.

Cao and Lai (2007) presented a genetic algorithm to tackle the shortcomings of premature and slow convergence of classical Genetic Algorithm (GA). Their research shows that the results and performance of the proposed method is better than the traditional genetic applications. Martinovic et al. (2009) proposed an adjustable simulated annealing with random initial solution algorithm to resolve single-commodity vrp with pickup and delivery service. They show that their results are good for different number of instances in practical applications. Caldas et al. (2009) presented a solution to the pickup and delivery routing problem with time windows by using tabu search algorithm. They stat that this algorithm is able to reduce the computational steps needed to solve the problem.
Time dependent VRP: In Time Dependent Vehicle Routing Problem (TDVRP), the travel time of a day is considered in addition to the travel time between two nodes (city or customer), taking into consideration the effect of the rush hours and the traffic congestion or any other delay on the vehicle's travel times. Xuping presented a disruption management model and an improved GA to resolve the VRP with disruption events that may happen during the routes such as traffic accidents or vehicle breakdowns. The model is based on a series of solving simplified strategies based on the theory of disruption management. These strategies are used to simplify the solution of complicated optimization problem and simplify the solution space. When a vehicle on a scheduled task in a distribution system breaks down, the other transport vehicles or additional ones could compensate for that and complete the mission to deliver the goods of the disabled vehicle at the time when serving the customer at the time is the most important goal for each solution. They improved the population and crossover operations in the genetic algorithm to improve the solution. They also improved the validity of strategies and algorithms by representing the disruption times and breakdown vehicles (Fig. 5).

Kok et al. (2012) presented a modified Dijkstra algorithm and a restricted dynamic programming heuristic to avoid the effect of congestion. A group of VRP instances were developed on actual network path, besides a fast model that considers the main factors of top time traffic congestion. They used the algorithm to solve the instances by avoiding different stages of congestion. 
Evolutionary algorithms: Evolutionary Algorithms (EAs) are a group of optimization techniques inspired by nature which uses natural operations such as selection and reproduction (crossover and mutation) in order to iteratively filter a set (population) of solution candidate's $\mathrm{x} \in \mathrm{X}$ from the search space $\mathrm{X}$ (Baldacci et al., 2004; Shaw, 1998). Their aim is to find the element(s) $x \in X$ for which the objective function $\mathrm{f}: \mathrm{X} \rightarrow \mathrm{R}$ takes on the optimal values. Evolutionary methods are called Multi-objective Evolutionary Algorithms (MEAn) when they involve multiple such functions $F=\left\{\mathrm{f}_{1}, \mathrm{f}_{2}, \ldots, \mathrm{f}_{\mathrm{n}}\right\}$.

All EAs are implemented according to the stages illustrated in the schema (Fig. 5). First an initial population is created from random individuals and then this population is evaluated by computing objective functions for them. Then, a fitness result is inserted for each individual in the population based on the evaluation results. These fitness values are the selection measure of the most promising individuals for reproduction process. The solution candidates are then reproduced via crossover and mutation operations. Subsequently, the cycle begins to repeat for the next generation to complete the operation (Weise, 2009). A competitive selection is performed iteratively to select the best solutions and remove the poor ones.

The high fitness solutions are recombined (crossover) with other solutions by exchanging parts of a solution with another. Mutation is performed for solution by changing a single element of the solution. Crossover and mutation are utilized to reproduce new solutions till the optimal or at least approximate solution is obtained (Fig. 6 and 7).

\section{Algorithm pseudo-code of EA (Bouhafs et al., 2010):} BEGIN

INITIALISE population with random candidate solutions;

EVALUATE each candidate; REPEATUNTIL (TERMINATION CONDITION is satisfied) DO:

- SELECT parents

- RECOMBINE pairs of parents

- MUTATE the resulting offspring

- EVALUATE new cadidates

- SELECT individuals for the next generation END DO

An extended discussion of issues involved in the implementation and use of evolutionary algorithms is included in many applications. Many techniques of EA are improved independently such as Evolutionary Programming (EP) that focuses on developing the functions genetic algorithm that focuses on enhancing solutions to huge problems, Genetic Programming (GP) that focuses on improving programs and Evolutionary

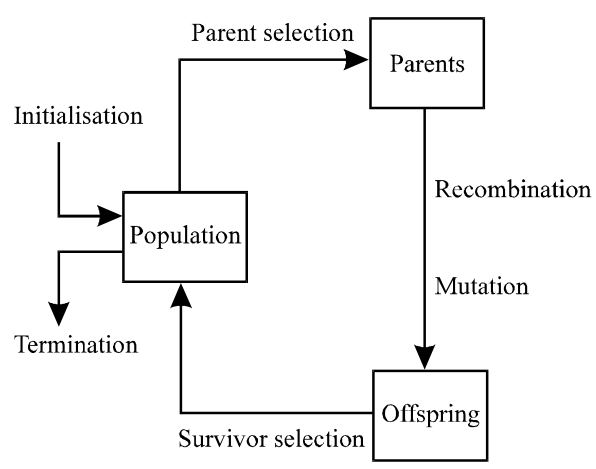

Fig. 6: The general scheme of an EA as a flow-chart

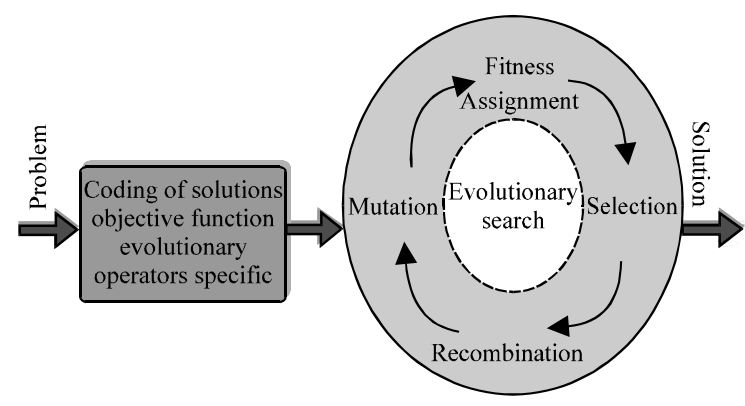

Fig. 7: Problem Solution using EA

Strategies (ES) that focuses on continuous development of recombination of functions (Zitzler and Thiele, 1999; Mohammed, 2015a, b; Potvin, 2007).

EA implementation: As usual to get good solutions an initial random population is formed in which the fitness function is entered with their offspring's. Higher fitness parents are selected for the next generation. The parents reproduce offspring's by performing crossover and mutation processes: crossover recombines the genes of the parent and mutation makes a slight random change to the individual genotype.

The theory to get the best results includes survival of the fittest based on competition between the old ones and new candidates to move to the next generation. These sequences of processes are repeated until a suitable solution is found or termination condition is reached (Whitley, 1994; Pop and Chira, 2014; Back, 1996). We represent a problem solution using evolutionary algorithms as shown in Fig. 8.

Evolutionary algorithm techniques: Many techniques have different implementation based on the nature of the problem (Celli et al., 2005; Mahdi et al., 2012).

Genetic algorithm: It is the famous type of EA techniques. GA is used in optimization and complex 


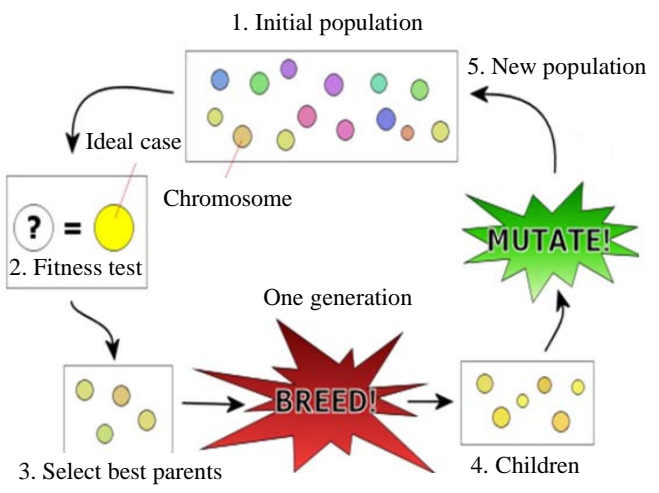

Fig. 8: Complete cycle of the GA

problems. It seeks the solution to a problem by applying operators such as crossover and mutation, etc.

Genetic programming: This type gets the solutions from computer programs. The fitness function determines the ability to solve and enhance a computational problem.

Evolutionary programming: This type is the same as genetic programming but the framework and structure of the program is fixed and mathematical models can be improvement.

Evolution strategy: It works on representations of solutions with real numbers and inspires the uses based on mutation rates.

Neuroevolution: It is the same as genetic programming but includes a representation for artificial neural networks by describing the framework, structure, parameters and connection weights.

\section{Genetic Algorithm (GA) theory and issues}

GA definition: Genetic Algorithm (GA) is an adaptive heuristic search algorithm in light of natural choice in this present reality where parents are chosen to create children who frame the populaces. Children with better qualities have a decent opportunity to survive, while children with less properties will vanish. The British naturalist Charles Darwin in 1859 proposed the theory of natural selection which stimulated John Holland to innovate the GA) in 1970 's (Mushtaq et al., 2015). Genetic algorithm imitates the natural selection process by using many techniques to generate new solutions to get approximate solution of a given problem. In GA, each individual (parent or child) is represented by a chromosome that contains genes. In VRP, each route is represented by a chromosome and the genes are cities or customers. Alphabets, decimal and binary numbers can be used for coding a chromosome.

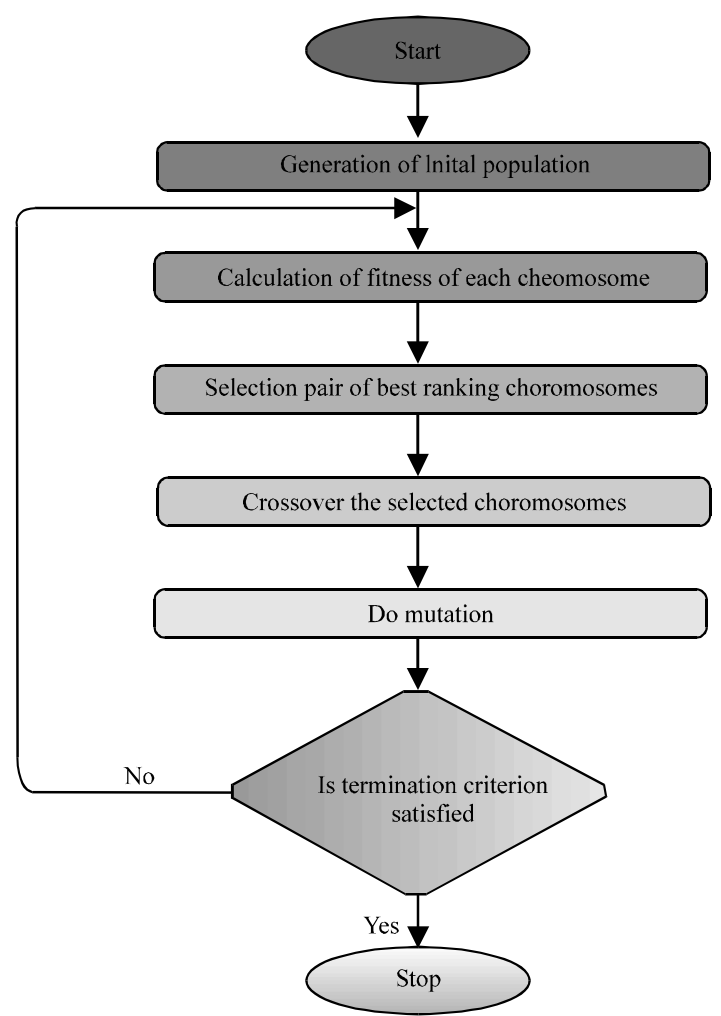

Fig. 9: The operations of general GA

Genetic algorithm in computer science aims to study the pre-written steps and create an artificial model to make them capable of solving problems which traditional computing approaches fail (Fonseca and Fleming, 1995). Figure 8 shows the general steps in the genetic algorithm.

GA operations: The basic functions in GA are population, crossover and mutation. The population (sets of chromosomes), contains all possible solutions. Chromosomes are selected according to a fitness value and each two are crossed over to generate new chromosomes. Mutation is used to make a change in the new chromosome to produce high quality chromosome until optimal or approximate solution is obtained. Figure 9 shows the flowchart for the genetic algorithms. The most important operators of the genetic algorithm are the crossover and mutation which can mainly affect the performance of the algorithm(Coello, 1999; Coello et al., 2007). A typical GA pseudo code is as follows:

- Select initial population

- Appraisal the individual fitness of a confirmed ratio of the population

- Repeat 


\begin{tabular}{ll} 
Table 1: Binary encoding & \\
\hline Genes & Coding \\
\hline Chromosome 1 & 1100010101010101 \\
Chromosome 2 & 1110011000010001 \\
\hline
\end{tabular}

Table 2: Permutation encoding

\begin{tabular}{ll}
\hline Genes & Coding \\
\hline Chromosome 1 & 126894357 \\
Chromosome 2 & 968714523 \\
\hline
\end{tabular}

- Choose pairs of good-ranking individuals to recreate

- Use crossover process

- Use mutation process

- Insert the pair of individuals in the new population

- Evaluate each individual in the new population

- Until terminating condition

Population: GA generally starts with some accepted solutions which is called the initial population. Each solution is represented by a chromosome. The initial population in general is generated randomly to create all possible solutions but this takes the algorithm a long time to find an approximate solution. Hence, it is recommended to generate part of the population by using a heuristic or by applying constraints in order to make the algorithm take less time to find the solutions (Bjarnadottir, 2004; Mohammed et al., 2016a, b).

Encoding: The chromosome contains a set of genes which contain information about the problem. Each gene represents the problem's variable characteristics. Chromosome representation is cold encoding which must get more attention when using GA to solve a problem. In many cases, encoding depends on the problem. In general, there are many techniques to represent the chromosomes (Neill et al., 2010; Pisinger and Ropke, 2005).

Binary encoding: Each chromosome is represented by one binary string in which each bit represents some characteristic of the solution. It is the common encoding but not for all problems. Michigan University Researchers are the first who used this coding for GA and becomes basic for many researches (Table 1 ).

Permutation encoding: In this encoding, numbers are assigned to the chromosome which is useful in direct problems such as the travelling sales man problem. This encoding is generally utilized in ordering issues in which some crossover and mutation are right to keep the chromosome values (Table 2).

Value encoding: String of some values related to the problem is assigned to each chromosome. The values can be numbers, real numbers or characters and others which
Table 3: Value encoding

\begin{tabular}{ll}
\hline Genes & Coding \\
\hline Chromosome 1 & 1.232 .168 .494 .67 \\
Chromosome 2 & Up, down, left, right \\
\hline
\end{tabular}

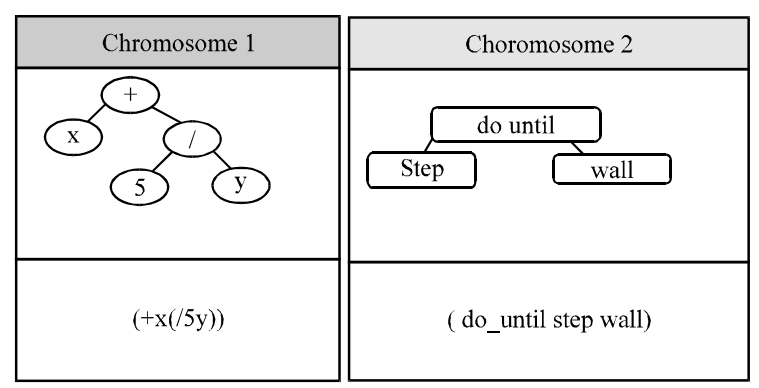

Fig. 10: Tree encoding

are useful to represent special problems. New crossover and mutation may be developed when using this encoding (Table 3 ).

Tree encoding: Used for devolving programs or expressions. Some objects such as programming instruction or function represent each chromosome (Goldberg and Holland, 1988) (Fig. 10).

Selection: The acceptable solutions are chosen from the first population or iteration when good fitness values are used and the solutions are mutated to form a new population which is used in the next iteration of the GA. Selection can be represented as follows (Miller and Goldberg, 1995), if we have set of population $\mathrm{P}=\left\{\mathrm{p}_{1}, \mathrm{p}_{1}\right.$, $\left.\ldots p_{n}\right\}$, select the $K$ best value SELECT $(p, k)$ of $P$, based on the fitness function. There are many selection methods for example, roulette wheel selection, rank selection, tournament selection, steady state selection, boltzmann selection and others.

Roulette wheel selection: It is the most common type of selection. The fitness values dominate the selection of the chromosome. The highest chromosomes fitness value has more chances to be selected.

Rank selection: Roulette selection can cause problems when there is considerable variation between the fitness values. If a chromosome has high fitness other chromosomes have less chances to be selected (Zhao et al., 2008) (Fig. 11). In this selection, the chromosomes are firstly ranked and then fitness from this ranking is assigned to each one. Fitness 1 assigned to the first worst one, fitness 2 assigned to the second worst, etc. and the best will have fitness $\mathrm{N}$ (number of chromosomes in population) (Fig. 12). 


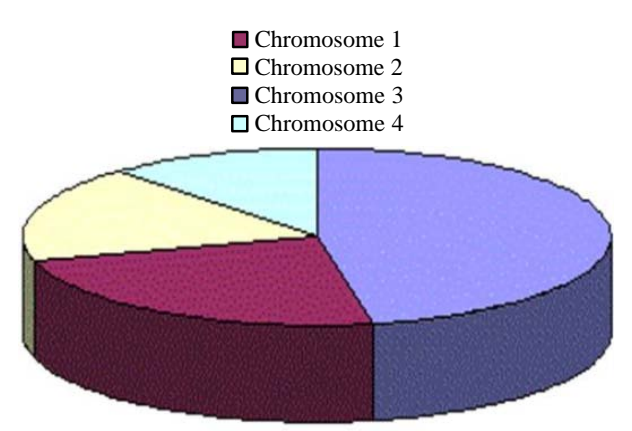

Fig. 11: Roulette selection

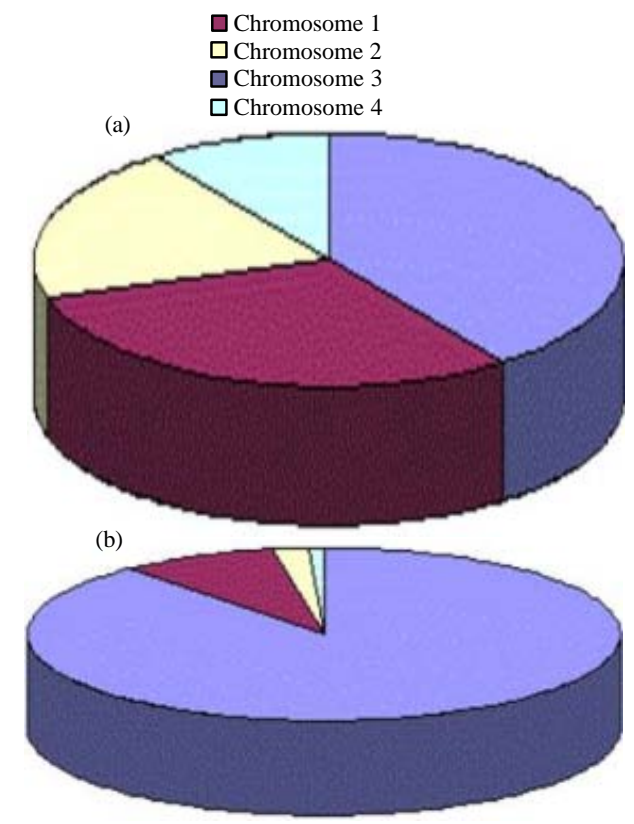

Fig. 12: Ranking selection: a) Before ranking and b) After ranking

Tournament selection: This includes working many tournaments through a few chromosomes selected randomly from the population operation. The crossover chooses the one which has the best fitness to get the best value. The tournament size adjusts the selection intensity. If the size is large for the tournament, a smaller chance for weak chromosomes to be selected. In a tournament, a random value between zero and one is generated and compared to a pre-determined selection probability to select the chromosomes. The weaker chromosome is chosen only if the random value is more than the selection probability (Man et al., 1996; Aggarrwal et al., 1997). The pseudo code of tournament selection as follows:

- Choos $\mathrm{K}$ (the tourmament size) individuals from the population ar random

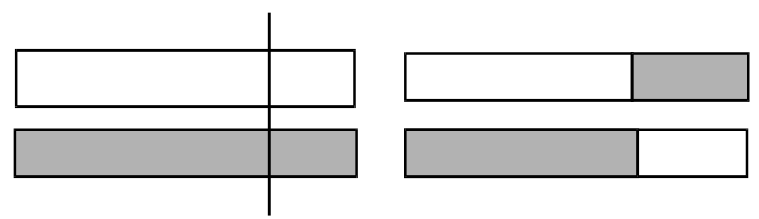

Fig. 13: Single point crossover
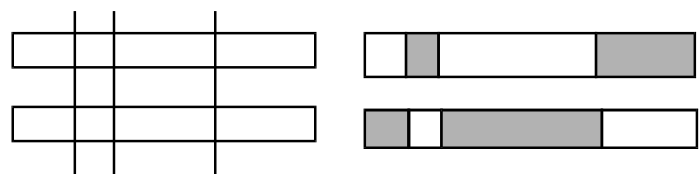

Fig. 14: Multi point crossover

- Choose the best individuals from pool toumament with probability $\mathrm{p}$

- Choose the second best individual with probability $\mathrm{p}^{*}(1-\mathrm{p})$

- Choose the third best individual with probability $\mathrm{p}^{*}\left((\mathrm{lp})^{2}\right)$

Crossover: It is the process of recombination between two chromosomes which are selected based on their fitness values to reproduce new chromosomes by exchanging portion of the two selected chromosomes. Random points are chosen along the bit string of the chromosome to do the exchange and the values of the two strings are exchanged according to these points. There are two types of crossovers: single point and multipoint (Chunhua, 2010) (Fig. 14 and 13).

Fitness value: $A$ fitness value represents the value of each solution in the population. It is evaluated by known function or by specially designed function. The GA uses this value to select the chromosome for reproduction. Fitness evaluation checks the solution value of each chromosome attributed by the objective function. Usually, this value varies across a range of problem domains and researchers generally normalized the fitness range from $0-1$. This normalized value represents the fitness of the individual chromosome which is exploited by the selection mechanism.

When an existing population is evaluated only fit solutions are kept to form the new population and unfit ones are eliminated. Such process enables the reproduction of new offspring's with good solutions. The fitness function plays an important role in improving the quality of the solutions.

Mutation: The process of mutation happens after the crossover process and before the offspring is released 
Table 4: Mutation

\begin{tabular}{ll}
\hline Genes & Coding \\
\hline Original choromosome 1 & 1101111000011110 \\
Original choromosome 2 & 1101100100110110 \\
Mutated choromosome 1 & 1100111000011110 \\
Mutated choromosome 2 & 1101101100110110 \\
\hline
\end{tabular}

into the solution space. The mutation operation introduces a random change on the sequence of the gene in the chromosome. Mutation depends on the encoding as well as the crossover. In permutation encoding, mutation could occur by exchanging two genes. In binary encoding, a few randomly chosen bits from 1-0 or from 0-1 can be switched (Hashi et al., 2015). A mutation scheme determines whether the mutation process will benefit the overall fitness of the chromosome with the specified constraints. The mutation process only occurs when the chromosomes are rewarded or benefited.

Many different mutation schemes are used such as gaussian mutation, uniform mutation, boundary mutation, dynamic mutation, insertion mutation, swap mutation displacement mutation and non uniform mutation (Table 4).

\section{CONCLUSION}

Genetic algorithm is efficient in solving highly complex computational problems such as VRP. These are mainly optimization problems. It is efficient to (Mohammed et al., 2016a, b; Powell et al., 2001; Caldas et al., 2009):

- Find a solution when the solution space is large and when linear programming method is unable to find theoretical solution in proper time

- Deal with multi-constraints problems

- Solve a problem when there is limited time or resources of the problem

- Find approximate solution

- Handle a problem when there is no exact or known algorithm exists to solve it

- In distributing a problem on parallel computers which is difficult by linear programming, the distributed results can be easily compared

- Stop the execution at any time because there is a solution at any time, whatever it might be, better or worse

Genetic algorithms are non-deterministic, i.e., they are stochastic in decisions which make them more robust (Zhang and Li, 2014). It is a key technology in random search to solve unclear and complex problems which require large time space for optimal solution. It is an important technique in the search for the perfect choice of a solution set that is available for a particular design. Genetic algorithm is an excellent technique to solve huge problems with a high computational complexity, especially in computer science where the problems need to enhance solutions. GA can be applied in the following applications:

- Tour selection, the goal of tour optimization for travelling salesman problem and vehicle routing problem to reduce the costs and visit all locations with constraints

- With Neural Networks in which the operation of pattern selection or classification based on a previous input to select optimal pattern

- Multi-constrained problems such as routing of the network for the internet with different features

- Many types of optimization problems such as Distributing packages, traffic optimization, puzzle optimization and others

The trend begins using heuristic, especially Genetic Algorithm (GA) to find a good solution for complex problems, particularly, when other search techniques fail to find an optimal solution. GA is a key technology in random search to solve unclear and complex problems which require large time space for optimal solutions. It is an important technique in the search for the perfect choice of a solution set that is available for a particular design. Exact algorithms such branch-and-cut-and-price can only solve relatively small problems but combining the classical branch-and-cut and pricing methods manifest new and good ideas. For the heuristics, a number of approximate algorithms have produced acceptable results for large complex problems. Tabu search, fuzzy logic, simulated annealing and the hybrid ones are used by many researchers to find approximate solutions in proper running times. Now a days, evolutionary algorithms like Genetic algorithm and neural network are the main interests of many researchers. GA may not solve the problem and find an optimal solution or may lead to the dead end (Bjarnadottir, 2004) but it can find an acceptable or feasible solution better than other algorithms in reasonable time.

Methodologies like Genetic Algorithms (GAs), Evolutionary Algorithms (EAs), etc. have been applied to achieve optimum solutions with mixed success. There are many techniques in solving examination timetable problems, some looks at the general or overall algorithm and some looks at part of the processes. However, all of the studies aim at improving the quality and effectiveness of GAs. Genetic Algorithm effectively demonstrates the ability to solve complex optimization problems. 
This study has attempted to comprehensively review the literature on one of the most researched problems which is the vehicle routing problem. It has outlined the theoretical and practical background for the study of the problem, demonstrated the importance of this study and presented many examples on the use of genetic algorithm in finding optimal solutions to the problems of vehicle routing.

\section{ACKNOWLEDGEMENT}

This research has been funded and supported by fellowship scheme (UTeM Zamalah scheme) by Universiti Teknikal Malaysia Melaka, Malaysia

\section{REFERENCES}

Aggarrwal, C., J.B. Orlin and R.P. Tai, 1997. Optimized crossover for the independent set problem. Operat. Res., 45: 226-234.

Azi, N., M. Gendreau and J.Y. Potvin, 2010. An exact algorithm for a vehicle routing problem with time windows and multiple use of vehicles. Eur. J. Oper. Res., 202: 756-763.

Back, T., 1996. Evolutionary Algorithms in Theory and Practice. 1st Edn., Oxford University Press, New York, USA., ISBN-13: 978-0195099713, pp: 328.

Baldacci, R., E. Hadjiconstantinou and A. Mingozzi, 2004. An exact algorithm for the capacitated vehicle routing problem based on a two-commodity network flow formulation. Oper. Res., 52: 723-738.

Barbucha, D. and P. Jedrzejowicz, 2007. An agent-based approach to vehicle routing problem. Intl. J. Appl. Math. Comput. Sci., 4: 18-23.

Bjarnadottir, A.S., 2004. Solving the vehicle routing problem with genetic algorithms. Masters Thesis, Technical University of Denmark Kongens Lyngby, Denmark.

Bouhafs, L., A. Hajjam and A. Koukam, 2010. A hybrid heuristic approach to solve the capacitated vehicle routing problem. J. Artif. Intell. Theory Appl., 1: $31-34$.

Braysy, O. and M. Gendreau, 2001. Genetic algorithms for the vehicle routing problem with time windows. Arpakannus, 1: 33-38.

Caldas, A., L. Carpente and L.S. Freire, 2009. A reactive tabu search algorithm for the pickup and delivery routing problem with time windows. MSc Thesis, University of A Coruna, A Coruna, Spain.

Cao, E.B. and M.Y. Lai, 2007. Vehicle routing problem with simultaneous delivery and pick-up with time windows. Proceedings of the International Conference on Transportation Engineering, July 22-24, 2007, Southwest Jiaotong University, Chengdu, China, pp: 160-166.
Carlsson, J., D. Ge, A. Subramaniam, A. Wu and Y. Ye, 2009. Solving min-max multi-depot vehicle routing problem. Lectures Global Optim., 55: 31-46.

Celli, G., E. Ghiani, S. Mocci and F. Pilo, 2005. A multiobjective evolutionary algorithm for the sizing and siting of distributed generation. IEEE Trans. Power Syst., 20: 750-757.

Chunhua, T., 2010. An improving genetic algorithm for vehicle routing problem with time windows. Proceedings of the 2010 International Conference on Intelligent Computation Technology and Automation, Vol. 1, May 11-12, 2010, IEEE, Changsha, China, ISBN:978-1-4244-7280-2, pp: 603-606.

Coello, C.A.C., 1999. A comprehensive survey of evolutionary-based multiobjective optimization techniques. Knowledge Inform. Syst., 1: 269-308.

Coello, C.A.C., G.B. Lamont and D.A. Van Veldhuizen, 2007. Evolutionary Algorithms for Solving Multi-Objective Problems. 2nd Edn., Springer, New York, USA., ISBN-13: 9780387332543 , Pages: 800.

Cordeau, J.F. and M. Gendreau, 2002. Tabu search heuristics for the vehicle routing problem. Canada Research Chair in Distribution Management and GERAD, Canada.

Cortes, P., G.R.A. Montoya, J. Munuzuri and C.A. Espinal, 2017. A tabu search approach to solving the picking routing problem for large-and medium-size distribution centres considering the availability of inventory and $\mathrm{K}$ heterogeneous material handling equipment. Appl. Soft Comput., 53: 61-73.

Csiszar, S., 2005. Route elimination heuristic for vehicle routing problem with time windows. Acta Polytech. Hungarica, 2: 77-89.

Desaulniers, G., P.E. Gagnon and L.M. Rousseau, 2009. A large neighborhood search algorithm for the vehicle routing problem with time windows. Networks, 54: 190-204.

Desrochers, M., C.V. Jones, J.K. Lenstra, M.W. Savelsbergh and L. Stougie, 1999. Towards a model and algorithm management system for vehicle routing and scheduling problems. Decis. Support Syst., 25: 109-133.

Desrochers, M., J.K. Lenstra and M.W. Savelsbergh, 1990. A classification scheme for vehicle routing and scheduling problems. Eur. J. Oper. Res., 46: 322-332.

Eksioglu, B., A.V. Vural and A. Reisman, 2009. The vehicle routing problem: A taxonomic review. Comput. Ind. Eng., 57: 1472-1 483. 
Feng, Z.W., H.D. Lian, J.H. Chao and G.L. Xia, 2009. An improved genetic algorithm for vehicle routing problem of non-full load. Proceedings of the 3rd International Symposium on Intelligent Information Technology Application, Vol. 2, November, 21-22, 2009, IEEE, Shijiazhuang, China, ISBN:978-0-7695 -3859-4, pp: 173-175.

Fonseca, C.M. and P.J. Fleming, 1995. An overview of evolutionary algorithms in multiobjective optimization. Evol. Comput., 3: 1-16.

Goel, A. and V. Gruhn, 2006. Solving a dynamic real-life vehicle routing problem. Oper. Res. Proc., 2005: 367-372.

Goldberg, D.E. and J.H. Holland, 1988. Genetic algorithms and machine learning. Mach. Learn., 3: 95-99.

Hashi, E.K., M.R. Hasan and M.S.U. Zaman, 2015. A heuristic solution of the vehicle routing problem to optimize the office bus routing and scheduling using clarke and wright's savings algorithm. Proceedings of the 1st International Conference on Computer and Information Engineering, November 26-27, 2015, IEEE, Dhaka, Bangladesh, ISBN:978-1-4673-8343-1, pp: 13-16.

Kok, A.L., E.W. Hans and J.M.J. Schutten, 2012. Vehicle routing under time-dependent travel times: The impact of congestion avoidance. Comput. Oper. Res., 39: 910-918.

Kovacs, A., 2008. Solving the vehicle routing problem with genetic algorithm and simulated annealing. Master Thesis, Dalarna University, Falun, Sweden.

Kuske, S. and M. Luderer, 2010. Autonomous units for solving the capacitated vehicle routing problem based on ant colony optimization. Electron. Commun. EASST., 26: 1-23.

Laporte, G., 1992. The vehicle routing problem: An overview of exact and approximate algorithms. Eur. J. Oper. Res., 59: 345-358.

Laporte, G., 2009. Fifty years of vehicle routing. Transp. Sci., 43: 408-416.

Lau, H.C., T.M. Chan, W.T. Tsui and W.K. Pang, 2010. Application of genetic algorithms to solve the multidepot vehicle routing problem. IEEE. Trans. Autom. Sci. Eng., 7: 383-392.

Lopes, H.S., M.V.L. Dalle and C.R.E. Lima, 2005. An ant colony optimization system for the capacitated vehicle routing problem. Proceedings of the XXVI Iberian Latin-America Congress on Computational Methods in Engineering CILAMCE 2005, October 19-21, 2005, ABMEC \& AM, Espirito Santo, Brazil, pp: $1-12$.

Mahdi, O.A., M.A. Mohammed and A.J. Mohamed, 2012. Implementing a novel approach an convert audio compression to text coding via hybrid technique. Intl. J. Comput. Sci. Issues, 9: 53-59.
Man, K.F., K.S. Tang and S. Kwong, 1996. Genetic algorithms: Concepts and applications in engineering design. IEEE. Trans. Ind. Electron., 43: 519-534.

Martinovic, G., I. Aleksi and A. Baumgartner, 2009. Single-commodity vehicle routing problem with pickup and delivery service. Math. Prob. Eng., 2008: $1-17$.

Miller, R.L. and D.E. Goldberg, 1995. Genetic algorithms, tournament selection and the effects of noise. Complex Syst., 9: 193-212.

Mohammed, M.A., 2015a. Design and implementing an efficient expert assistance system for car evaluation via fuzzy logic controller. Intl. J. Comput. Sci. Software Eng., 4: 60-68.

Mohammed, M.A., 2015b. Investigating role of knowledge auditing in profile of the business unit Information Technology and Computer Center (ITCC) University of Anbar. Intl. J. Enhanced Res. Manage. Comput. Appl., 4: 10-18.

Mohammed, M.A., A.B. Khateeb and D.A. Ibrahim, 2016a. Case based reasoning shell frameworkas decision support tool. Indian J. Sci. Technol., Vol. 9, 10.17485/1jst/2016/v9i42/101280

Mohammed, M.A., A.K. Belal and D.A. Ibrahim, 2016 b. Human interaction with mobile devices on social networks by young and elderly people: Iraq a case study. Indian J. Sci. Technol., Vol. 9, 10.17485/ijst/ 2016/v9i42/101281.

Mohammed, M.A., A.T.Y. Aljumaili and H.A. Salah, 2014. Investigation the role of cloud computing in the business value for optimal criteria. Intl. J. Enhanced Res. Sci. Technol. Eng., 3: 111-118.

Mohammed, M.A., M.S. Ahmad and S.A. Mostafa, 2012. Using genetic algorithm in implementing capacitated vehicle routing problem. Proceedings of the 2012 International Conference on Computer and Information Science (ICCIS), June 12-14, 2012, IEEE, Ramadi, Malaysia, ISBN:978-1-4673-1937-9, pp: 257-262.

Mohammed, M.A., O.I. Obaid and M.S. Ahmad, 2015. Using Genetic Algorithm in Solving Capacitated Vehicle Routing Problem. OmniScriptum Publishing, Saarbrucken, Germany,

Montemanni, R., L.M. Gambardella, A.E. Rizzoli and A.V. Donati, 2003. A new algorithm for a dynamic vehicle routing problem based on ant colony system. Proceedings of the Second International Workshop on Freight Transportation and Logistics, April 18-20, 2003, Istituto Dalle Molle Di Studi Sull Intelligenza, Manno, Switzerland, pp: 27-30.

Mostafa, S.A., M.S. Ahmad and M. Firdaus, 2012. A soft computing modeling to case-based reasoning implementation. Intl. J. Comput. Appl., 47: 14-21. 
Mushtaq, A.D., M. Hojabri, D. Hamdan and M.H. Ali, 2015. Maximum power prediction for PV system based on $\mathrm{P}$ and $\mathrm{O}$ Algorithm. J. Adv. Appl. Sci., 3: 113-118.

Nazif, H. and L.S. Lee, 2010. Optimized crossover genetic algorithm for vehicle routing problem with time windows. Am. J. Applied Sci., 7: 95-101.

Neill, O.M., L. Vanneschi, S. Gustafson and W. Banzhaf, 2010. Open issues in genetic programming. Genetic Program. Evolvable Mach., 11: 339-363.

Obaid, O.I, M.A. Mohammed and M.S. Ahmad, 2015. Solving Examination Timetabling Problem by using Genetic Algorithm. OmniScriptum Publishing, Saarbrucken, Germany,.

Obaid, O.I., M. Ahmad, S.A. Mostafa and M.A. Mohammed, 2012. Comparing performance of genetic algorithm with varying crossover in solving examination timetabling problem. J. Emerg. Trends Comput. Inf. Sci., 3: 1427-1434.

Ozyurt, Z., D. Aksen and N. Aras, 2006. Open Vehicle Routing Problem with Time Deadlines: Solution Methods and an Application. In: Operations Research Proceedings, Haasis, H.D., H. Kopfer and J. Schonberger (Eds.). Springer, Berlin, Germany, pp: 73-78.

Parragh, S.N., K.F. Doerner and R.F. Hartl, 2008. A survey on pickup and delivery problems. J. Bus. Manage., 58: $21-51$.

Pisinger, D. and S. Ropke, 2005. A general heuristic for vehicle routing problems. Comput. Operat. Res., 34: 2403-2435.

Pisinger, D. and S. Ropke, 2005. A general heuristic for vehicle routing problems. Comput. Operat. Res., 34: 2403-2435.

Polacek, M., R.F. Hartl, K. Doerner and M. Reimann, 2004. A variable neighborhood search for the multi depot vehicle routing problem with time windows. J. Heuristics, 10: 613-627.

Pop, P. and C. Chira, 2014. A hybrid approach based on genetic algorithms for solving the clustered vehicle routing problem. Proceedings of the 2014 IEEE Congress on Evolutionary Computation, July 6-11, 2014, IEEE, Cluj-Napoca, Romania, ISBN:978-14799-1488-3, pp: 1421-1426.

Potter, T. and T. Bossomaier, 1995. Solving vehicle routing problems with genetic algorithms. Proceedings of the IEEE International Conference on Evolutionary Computation, Vol. 2, November 29-December1, 1995, IEEE, Canberra, Australia, ISBN:0-7803-2759-4, pp: 788-793.

Potvin, J.Y., 2007. Evolutionary Algorithms for Vehicle Routing. CIRRELT, Montreal, Quebec, Pages: 50.
Powell, W.B., J.A. Shapiro and H.P. Simao, 2001. A representational paradigm for dynamic resource transformation problems. Annal. Oper. Res., 104: 231-279.

Rizzoli, A.E., F. Oliverio, R. Montemanni and L.M. Gambardella, 2004. Ant colony optimisation for vehicle routing problems: From theory to applications. Bimonthly Cult. Exhibition Gallery, 9: $1-50$.

Ropke, S., 2005. Heuristic and exact algorithms for vehicle routing problems. P.hD Thesis, Computer Science Department, University of Copenhagen, Copenhagen, Denmark.

Sen, A. and K. Bulbul, 2008. A survey on multi trip vehicle routing problem. Proceedings of the International Congress on Logistics and Supply Chain, November 6-7, 2008, Sabanci University, Istanbul, Turkey, pp: $1-7$.

Shaw, P., 1998. Using constraint programming and local search methods to solve vehicle routing problems. Proceedings of the 4th International Conference on Principles and Practice of Constraint Programming, October 26-30, 1998, Pisa, Italy, pp: 417-431.

Tang, K.S., K.F. Man, S. Kwong and Q. He, 1996. Genetic algorithms and their applications. IEEE Signal Process. Magazine, 13: 22-37.

Torres, M.J.R., A.E.H. Lizarazo, E.G. Franco and A.X. Halabi, 2009. Using randomization to solve the deterministic single and multiple vehicle routing problem with service time constraints. Proceedings of the Conference on Winter Simulation, December 13-16, 2009, ACM, New York, USA., pp: 2989-2994.

Toth, P. and D. Vigo, 2002. Vehicle Routing Problem. In: SIAM Monographs on Discrete Mathematics and Applications, Toth, P. and D. Vigo (Eds.). SIAM, Philadelphia, Pennsylvania, pp: 157-194.

Toth, P. and D. Vigo, 2014. Vehicle Routing: Problems, Methods and Applications. 2nd Edn., Society for Industrial and Applied Mathematics, Philadelphia, Pennsylvania, ISBN:978-1-61197-358-7,.

Vidal, T., T.G. Crainic, M. Gendreau, N. Lahrichi and W. Rei, 2012. A hybrid genetic algorithm for multidepot and periodic vehicle routing problems. Oper. Res., 60: 611-624.

Vigo, D., 2007. Introduction to VRP. MSc Thesis, University of Bologna, Bologna, Italy.

Wang, X., X. Wu, Z. Wang and X. Hu, 2009. A model and an improved genetic algorithm for the vehicle routing problem with break-down vehicles. Proceedings of the 4th International Conference on Innovative Computing, Information and Control, December 7-9, 2009, IEEE, Dalian, China, ISBN:978-1-4244-5544-7, pp: 696-699. 
Weise, T., 2009. Global Optimization Algorithms-Theory and Application. 2nd Edn., Self-Publishing, USA., Pages: 455.

Whitley, D., 1994. A genetic algorithm tutorial. Statist. Comput., 4: 65-85.

$\mathrm{Xu}$, J., G. Goncalves and T. Hsu, 2008. Genetic algorithm for the vehicle routing problem with time windows and fuzzy demand. Proceedings of the International IEEE Congress on Evolutionary Computation and Computational Intelligence, June 1-6, 2008, IEEE, Arras, France, ISBN:978-1-4244-1822-0, pp: 4125-4129.

Yueqin, Z., L. JinfenG, D. Fu and R. Jing, 2007. Genetic algorithm in vehicle routing problem. Proceedings of the 3rd International Conference on Intelligent Information Hiding and Multimedia Signal Processing, Vol. 2, November 26-28, 2007, IEEE, Kaohsiung, Taiwan, pp: 1-3.
Zhang, J. and J. Li, 2014. A hybrid genetic algorithm to the vehicle routing problem with fuzzy cost coefficients. Proceedings of the 11th International Conference on Fuzzy Systems and Knowledge Discovery, August, 19-21, 2014, IEEE, Tianjin, China, ISBN:978-1-47995148-2, pp: 147-152.

Zhao, X., G. Goncalves and R. Dupas, 2008. A genetic approach to solving the vehicle routing problem with time-dependent travel times. Proceedings of the 16th Mediterranean Conference on Control and Automation, June 25-27, 2008, IEEE, Arras, France, ISBN:978-1-4244-2504-4, pp: 413-418. September 11, 2017

Zirour, M., 2008. Vehicle routing problem: Models and solutions. J. Qual. Meas. Anal., 4: 205-218.

Zitzler, E. andL. Thiele, 1999. Multiobjective evolutionary algorithms: A comparative case study and the strength pareto approach. IEEE Trans. Evol. Comput., 3: $257-271$. 